\title{
Using Quadratic Indexes in the Synthesis of Harmonic Disturbance Attenuation Compensators
}

\author{
M. Dilda and A. Piazzi \\ Dipartimento di Ingegneria dell'Informazione, \\ Università di Parma, Viale delle Scienze, I-43100 Parma, Italy \\ Phone: (39) 521-905733, Fax: (39) 521-905723, E-Mail: aurelio@ce.unipr.it
}

\begin{abstract}
The paper addresses the synthesis of fixed-structure compensators to attenuate an harmonic disturbance whose frequency is unknown but belongs to a given interval. Besides jointly employing the sensitivity function and Liénard-Chipard Criterion to give guaranteed stability margins, the main novelty of the proposed method lies in using (integral) quadratic indexes of unit step tracking error to shape time-domain performances. In such a way the compensator synthesis is converted into a new minimax optimization problem for which an approximate solution is attainable through known numerical algorithms. The method is applied to a problem arising in the control of wood contouring machines.
\end{abstract}

\section{INTRODUCTION}

In the context of linear time-invariant continuous systems consider the standard unity-feedback configuration of Fig. 1 where $P(s)$ and $C(s)$ are respectively the given rational transfer function of the plant and the compensator transfer function to be designed. Signals $y, r, e$, and $d$ are respectively the controlled output, the reference, the tracking error, and the harmonic disturbance modelled by a single sinusoid whose frequency is unknown but remains in the known range $\left[\omega^{-}, \omega^{+}\right]$.

Briefly stated the addressed problem is the following. Given any plant $P(s)$ (stable/unstable, minimum/nonminimum phase) design a fixed-structure compensator $C(s)$ such that: (i) the harmonic disturbance is asymptotically attenuated as much as possible for any frequency in the range $\left[\omega^{-}, \omega^{+}\right]$; (ii) closed-loop stability is achieved with guaranteed stability margins; (iii) timedomain performance specifications are given in terms of constraints on overshoot $\left(M_{p}\right)$, rise time $\left(T_{r}\right)$, and settling time $\left(T_{s}\right)$; (iv) the velocity error constant $K_{v}>0$ is assigned (type-one feedback system)

Specific motivation to this problem can be found in the control of wood contouring machines where fixedstructure compensators--such as PD controllers and low-order compensators - can be preferred for implementation easiness [1],[2].

Apart from trial-and-error design based on intensive computer simulations, which is not viable even for moderately complex compensators (such as those with three-four design parameters), a pertinent available design methodology is $H_{\infty}$ control, particularly suitable to include robustness specifications [3]. However, $H_{\infty}$ design may not yield the best result when performances involve overshoot, rise time, and settling time; moreover, the designed compensators may be of unnecessary high order [4]

In this paper it is shown how to resort to a minimax optimization problem to solve the posed synthesis problem. Instrumental to the optimization problem formulation is the substitution of time-domain specifications usually given as $M_{p} \leq \bar{M}_{p}, T_{r} \leq \bar{T}_{r}, T_{s} \leq \bar{T}_{s}$ with a set of constraints on integral quadratic indexes $J_{0} \leq \bar{J}_{0}, J_{1} \leq \bar{J}_{1}, J_{2} \leq \bar{J}_{2}$. These indexes are defined as $\bar{J}_{i}:=\int_{0}^{\infty}\left[D^{i} e(t)\right]^{2} \overline{d t}, i=0,1,2$, where $e(t)$ is the tracking error in response to unit step reference and $D^{i}$ indicates $i$ th-order derivative. Bar over symbols denote acceptable time-domain performances not to be downgraded.

Closed-form expressions and recursive algorithms to compute indexes $J_{i}$ are known in the literature [510]: the Appendix reports the determinantal method adopted in this work. It has been showed, by qualitative and quantitative reasoning, that imposing constraints on indexes $J_{i}$ implicitly yields specifications on classical time-domain performances $M_{p}, T_{r}$, and $T_{s}$ [11].

Paper's organization. Section II exposes the problem analysis which yields to a minimax optimization problem whose approximate solution is obtained through frequency discretization, A worked example, taken from motion control of a tool machine for furniture industry, is exposed in Section III. Conclusions are drawn in last Section IV.

Notation: Denote with $C(k ; s)$ the chosen fixedstructure compensator function, rational in all arguments, where $k=\left(k_{1}, k_{2}, \ldots, k_{p}\right) \in \Re^{p}$ is the design parameter vector. The characteristic polynomial of unityfeedback loop be $\xi(k ; s)=a_{0}(k) s^{n}+a_{1}(k) s^{n-1}+\ldots+$ $a_{n}(k)$. The Hurwitz determinant of order $i$ associated to $\xi(k ; s)$ is denoted by $\Delta_{i}(k)$.

The loop transfer function is given by $L(k ; s):=$ $C(k ; s) P(s)$ and the sensitivity function is $S(k ; s):=$ $1 /(1+L(k ; s))$. Denote also with $J_{i}(k), i=0,1,2$, the rational function of $k$ for which $J_{i}(k)=J_{i}$ provided that $\xi(k ; s)$ is (Hurwitz) stable. $J_{i}(k)$ can be computed as shown in the Appendix.

\section{FIXED-STRUCTURE COMPENSATOR \\ DESIGN VIA MINIMAX OPTIMIZATYON}

The design procedure begins by choosing $C(k ; s)$ the fixed-structure compensator tranfer function-for which the following assumptions are retained: 1) the 


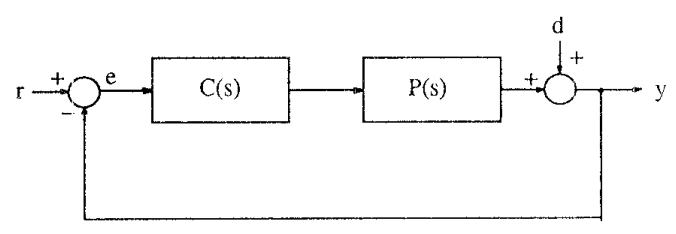

Fig: 1. Unity-feedback control system.

parameter vector $k$ belong to $\mathcal{B}$, a $p$-dimensional interval of $\Re^{p}$;2) The first coefficient of $\xi(k ; s)$ is always positive : $\left.a_{0}(k)>0 \forall k \in \mathcal{B} ; 3\right) \lim _{s \rightarrow 0} s L(k ; s)=$ $\lim _{s \rightarrow 0} s C(k ; s) P(s)=K_{v}>0 \quad \forall k \in \mathcal{B}$.

These assumptions are not to be considered restrictive since the interval $\mathcal{B}$ can be enlarged as much as necessary and any fixed-order compensator with prescribed zero-pole pattern (stable/unstable/critical) can always be parametrized to satisfy assumptions 2) and 3) (cf. next Section III).

Remark 1: Note how assumption 3) accounts for the required steady-state accuracy [point (iv) of specifications; cf. Section I].

The measure of asymptotic harmonic attenuation at, a given frequency is $|S(k ; j \omega)|$. Thus the design objective (i) lies in minimizing, over $\mathcal{B}, \max _{\omega \in\left[\omega^{-}, \omega^{+}\right]}|S(k ; j \omega)|$ which corresponds to posing a minimax synthesis problem.

The following lemma, which is a variant of LiénardChipard Criterion [12, pag. 221], is introduced. Its proof can be found in [11].

Lemma 1: Assume $a_{0}>0$. The polynomial $a_{0} s^{n}+$ $a_{1} s^{n-1}+\ldots+a_{n-1} s+a_{n}$ is (Hurwitz) stable if and only if:

$$
\begin{aligned}
& a_{n}>0, \Delta_{n-1}>0 \\
& a_{n-1} \geq 0, a_{n-3} \geq 0, \ldots, a_{l} \geq 0(l \geq 1), \\
& \Delta_{n-3}>0, \Delta_{n-5}>0, \ldots, \Delta_{q}>0(q \geq 2)
\end{aligned}
$$

Assumption 3) implies that $L(k ; s)=\chi(k ; s) /[s \psi(k ; s)]$ where $\chi(k ; s)$ and $\psi(k ; s)$ are suitable real polynomials of complex variable $s$, depending on $k$, with $\chi(k ; 0) \neq 0$ $\forall k \in \mathcal{B}$. Hence it follows that $\xi(k ; s)=s \psi(k ; s)+\chi(k ; s)$ and the Laplace transform of the unit step tracking error is given by $E(s)=\psi(k ; s) / \xi(k ; s)$. An immediate consequence of Lemma 1 and formula $J_{0}(k)=$ $D_{0}(k) /\left[a_{0}(k) a_{n}(k) \Delta_{n-1}(k)\right]$ (see formulae 19 and $20 \mathrm{in}$ the Appendix) is the next property which gives necessary and sufficient conditions for closed-loop stability.

Property 2: For a given $k \in \mathcal{B}$, the unity-feedback control system is (Hurwitz) stable if and only if ( $l \geq$ $1 ; q \geq 2)$ :

$$
\begin{array}{lr}
a_{n}(k) \geq 0, \Delta_{n-1}(k) \geq 0, & (2 c) \\
a_{n-1}(k) \geq 0, a_{n-3}(k) \geq 0, \ldots, a_{l}(k) \geq 0, & (2 b) \\
\Delta_{n-3}(k)>0, \Delta_{n-5}(k)>0, \ldots, \Delta_{q}(k)>0,(2 c) \\
J_{0}(k)<+\infty .
\end{array}
$$

Stability margins are introduced by imposing that, for a constant real $M>1$ :

$$
|1+L(k ; j \omega)| \geq \frac{1}{M} \quad \forall \omega \geq 0 .
$$

Provided that $\xi(k ; s)$ is stable, statement (3), interpreted on the Nyquist plot, implies that gain margin $G_{M}$ and phase margin $P_{M}$ satisfy:

$$
G_{M} \geq \frac{M}{M-1} ; \quad P_{M} \geq \cos ^{-1}\left(1-\frac{1}{2 M^{2}}\right) \text {. }
$$

The above expressions (4) constitute guaranteed minimal stability margins. Observe also that (3) is equivalent to

$$
|S(k ; j \omega)| \leq M \quad \forall \omega \geq 0 \text { or }\|S(k ; s)\|_{\infty} \leq M(5 a, b)
$$

with $\|\cdot\|_{\infty}$ denoting the $H_{\infty}$-norm of the sensitivity function [3]. When stability margin specifications are assigned as $G_{M} \geq \bar{G}_{M}$ and $P_{M} \geq \bar{P}_{M}$ the constant $\mathrm{M}$ can be chosen according to:

$$
\frac{1}{M} \geq \max \left\{1-\frac{1}{\bar{G}_{M}}, 2 \sin \frac{\bar{P}_{M}}{2}\right\} .
$$

Remark 2: The proposed method for assuring closedloop stability margins does not permit with certainty to impose $\bar{P}_{M} \geq 60^{\circ}$, but this is hardly a truly linitation since $60^{\circ}$ for phase margin is fairly high and formula (6) is, in pratice, rather conservative.

The set of time-domain performance specifications $T_{r} \leq \bar{T}_{r}, T_{s} \leq \bar{T}_{s}$, and $M_{p} \leq \bar{M}_{p}$ [point (iii) of specifications] is replaced with $\bar{J}_{0} \leq \bar{J}_{0}, J_{1} \leq \bar{J}_{1}$, and $J_{2} \leq \bar{J}_{2}$ with suitable values $\bar{J}_{i}$ to be found in the course of synthesizing the actual compensator through iterative use of a minimax optimization solver. The validity of such a substitution has been carefully examined in [11] where a theoretical analysis of time-domain performance relative to a second-order system, or a system with a pair of dominant poles, is exposed. We can add that using these quadratic indexes $J_{i}$ has been proved effective in all compensator syntesis examined during this research and it is reasonable to expect their usefulness in the broader field of optimization-based control system design. In particular it is emerged the sharp convenience, from the point of view of both the computational burden and time-domain specification effectiveness, of using indexes $J_{i}$ insted of root clustering regions [13].

The following minimax optimization problem can be considered to design an harmonic disturbance attenuation compensator.

Minimax Optimization Problem: Given finite timedomain performance indexes $\bar{J}_{0}, \bar{J}_{1}, \bar{J}_{2}$, and the upperbound $\mathrm{M}$ on sensitivity $H_{\infty}$-norm, find an optimal compensator $C\left(k^{*} ; s\right)$ as a solution to $(\epsilon>0 ; l \geq 1 ; q \geq 2)$ :

$$
\min _{k \in \mathcal{B}} \max _{\omega \in\left[\omega^{-}, \omega+\right]}|S(k ; j \omega)|
$$


subject to

$$
\begin{aligned}
& a_{n}(k) \geq 0, \Delta_{n-1}(k) \geq 0 \\
& a_{n-1}(k) \geq 0, a_{n-3}(k) \geq 0, \ldots, a_{l}(k) \geq 0 \\
& \Delta_{n-3}(k) \geq \epsilon, \Delta_{n-5}(k) \geq \epsilon, \ldots, \Delta_{q}(k) \geq \epsilon, \\
& J_{0}(k) \leq \bar{J}_{0}, \quad J_{1}(k) \leq \bar{J}_{1}, \quad J_{2}(k) \leq \bar{J}_{2} \\
& |S(k ; j \omega)| \leq M \quad \forall \omega \geq 0 .
\end{aligned}
$$

This optimization problem, which stems quite naturally from synthesis specifications, is a minimax nonlinear optimization problem with $n+4$ inequality constraints, one of which is the semi-infinite constraint ( $7 f$ ). In particular, note how problem (7) incorporates specifications on closed-loop stability margins throught Property 2 and functional inequality (5a).

With regard to inequalities $(7 \mathrm{~d})$, the positive number $\epsilon$ is introduced in order to impose that $\Delta_{i}(k) \quad i=$ $n-3, n-5, \ldots, p$ be strictly positive as required by Property 2. For example, $\epsilon$ can be any sufficiently small value compatible with computation precision of the adopted numerical algorithm to solve (7), but in pratice, it can always be set to zero. Indeed the following observations hold (cf. [11]):

a) In the case that $n \leq 6$, setting $\epsilon=0$ inequalities $(7 \mathrm{~b}, \mathrm{c}, \mathrm{d})$ with $J_{0}(k) \leq \overline{\bar{J}}_{0}$ if satisfied for some $k \in \mathcal{B}$ assure with certainty closed-loop stability.

b) In case that $n>6$, setting $\epsilon=0$ inequalities $(7 \mathrm{~b}, \mathrm{c}, \mathrm{d})$ with $J_{0}(k) \leq \bar{J}_{0}$ if satisfied for some $k \in \mathcal{B}$ assure with probability one, over the space of all compensator transfer functions $C(k ; s)$, closed-loop stability.

Remark 3: In (7) at the optimal solution $k^{*}$, the active constraints can only be found among inequalities (7e,f) and/or the boundaries of $B$. To know this could be useful in devising an ad hoc algorithmic procedure to solve (7).

Remark 4: The optimization problem (7) can exhibit various "conflicts" among design objectives. In particular it is known as waterbed effect [3, pag. 97], for nonminimum phase plant, the contention between minimizing $\max _{\omega \in[\omega-, \omega+]}|S(k ; j \omega)|$ and satisfyng $|S(k ; j \omega)| \leq$ $M \forall \omega \geq 0$ with $M$ not too large. In the present context of fixed-structure compensator synthesis, this conflict may even arise for minimum phase plants.

Remark 5: The reason of using stability constraints which are derived from Liénard-Chipard Criterion instead from the well-known Routh-Hurwitz Criterion is that the latter criterion requires computations of all the Hurwitz determinants whereas the former (approximately) requires only half of thern.

Exact determination of the optimal compensator $C\left(k^{*} ; s\right)$ is a difficul global optimization problem [14]. On the other hand, an approximate solution can be obtained recasting problem (7) into the following discrete minimax problem through a suitable discretization of the frequency axis.
Discrete Minimax Optimization Problem: Choose discretization frequencies in the range $\left[\omega^{-}, \omega^{+}\right]$and its complement: $\delta_{i} \in\left[\omega^{-}, \omega^{+}\right], \quad i=1,2, \ldots, m ; \eta_{i} \in$ $\left(0, \omega^{-}\right) \cup\left(\omega^{+},+\infty\right) i=1,2, \ldots, v$. Find a solution to $(l \geq 1 ; q \geq 2)$ :

$$
\min _{k \in \mathcal{B}} \max _{i=1, \ldots, m}\left\{\left|S\left(k ; j \delta_{i}\right)\right|\right\}
$$

subject to

$$
\begin{array}{lr}
a_{n}(k) \geq 0, \Delta_{n-1}(k) \geq 0, & (8 b) \\
a_{n-1}(k) \geq 0, a_{n_{-}-3}(k) \geq 0, \ldots, a_{l}(k) \geq 0, \quad(8 c) \\
\Delta_{n-3}(k) \geq 0, \Delta_{n-5}(k) \geq 0, \ldots, \Delta_{q}(k) \geq 0,(8 d) \\
J_{0}(k) \leq \bar{J}_{0}, \quad J_{1}(k) \leq \bar{J}_{1}, \quad J_{2}(k) \leq \bar{J}_{2}, \quad(8 e) \\
\left|S\left(k ; j \eta_{i}\right)\right| \leq M \quad(i=1, \ldots, v) .
\end{array}
$$

Solution to optimization problem (8) can be attained by means of sequential quadratic programming (SQP) $[15,16]$ for which an implementation is given by the "Matlab Optimization 'Toolbox" [17].

Caution, however, has to be used with any implementation of a SQP method to solve (8), since these methods can not guarantee convergence to a global solution.

\section{AN EXAMPLE}

\section{A. The Problem}

The electrical drive assigned to axis control of a wood contouring machine is characterized, disregarding highfrequency behaviour, by transfer function

$$
P(s)=g_{p} \frac{\omega_{n}^{2}}{s\left(s^{2}+2 \delta \omega_{n} s+\omega_{n}^{2}\right)}
$$

where $g_{p}=0.10\left[\mathrm{~ms}^{-1} \mathrm{~V}^{-1}\right], \omega_{n}=97[\mathrm{rad} / \mathrm{s}]$, and $\delta=$ 0.25 . Depending on tool machine operations this plant is subjected to a sinusoidal disturbance whose frequency is in the range $\left[\omega^{-}, \omega^{+}\right]$with $\omega^{-}=40[\mathrm{rad} / \mathrm{s}]$ and $\omega^{+}=80[\mathrm{rad} / \mathrm{s}]$.

It is required to synthesize a compensator in a unityfeedback control system which satisfies requirements (i)-(iv) (cf. Section I) with

$$
\begin{aligned}
K_{u} & =30\left[\mathrm{~s}^{-1}\right], \\
G_{m} & \geq 3, P_{m} \geq 40^{\circ}, \\
T_{r} & \leq 0.05[\mathrm{~s}], T_{s} \leq 1[\mathrm{~s}], M_{p} \leq 5 \% .
\end{aligned}
$$

B. The Fixed-structure Compensators

1) $\mathrm{PD}$ controller

Consider the PD controller (proportional-derivative) given by

$$
C_{1}(k ; s)=g_{c}\left(1+\frac{k_{1} s}{1+\left(k_{1} / N\right) s}\right)
$$

with $N$ the "noise" factor set to $30, k_{1}$ the time constant of the derivative action, and $g_{c}$ the static gain of the 
compensator. Here we simply have $k:=k_{1} \in \mathcal{B} \subseteq \Re$ and choose $\mathcal{B}:=\left[10^{-6}, 0.06\right]$.

2) thind-order compensator

Consider a third-order biproper compensator

$$
C_{2}(s)=A \frac{\left(s-z_{1}\right)\left(s-z_{2}\right)\left(s-z_{3}\right)}{\left(s-p_{1}\right)\left(s-p_{2}\right)\left(s-p_{3}\right)}
$$

with the following pole-zero pattern:

a) all zeros and poles lie in the open left half-plane;

b) all zeros and poles are real except two of the zeros which are conjugate complex: let they be $z_{2}$ and $z_{3}$. Then compensator (12) can be parametrized according to

$$
C_{2}(s)=C_{2}(k ; s)=g_{c} \frac{\left(1+k_{4} s\right)\left(1+2 k_{5} k_{6} s+k_{6}^{2} s^{2}\right)}{\left(1+k_{1} s\right)\left(1+k_{2} s\right)\left(1+k_{3} s\right)}
$$

with corresponding relations:

$g_{c}=C_{2}(0)=\left(A z_{1} z_{2} z_{3}\right) /\left(p_{1} p_{2} p_{3}\right), k_{i}=-\left(1 / p_{i}\right)>0$, $i=1,2,3, k_{4}=-\left(1 / z_{1}\right)>0, k_{5}=-\left(z_{2}+z_{3}\right) /\left(2 \sqrt{z_{2} z_{3}}\right)$, $k_{5} \in(0,1), k_{6}=1 / \sqrt{z_{2} z_{3}}>0$.

The compensator design vector is $k:=\left[k_{1} k_{2} k_{3} k_{4} k_{5} k_{6}\right]^{T}$ $\in \mathcal{B} \subseteq \Re^{6}$ with $\mathcal{B}:=\left[10^{-3}, 1\right] \times\left[10^{-3}, 1\right] \times\left[10^{-3}, 1\right] \times$ $\left[10^{-2}, 10\right] \times[0.1,0.9] \times[0.005,1]$.

\section{Computational Results}

For both the proposed compensators it is readily apparent that all the assumptions made in Section II can be satisfied. Indeed $a_{0}(k)=k_{1} / N>0 \forall k \in \mathcal{B}$ relative to PD controller (11) and $a_{0}(k)=k_{1} k_{2} k_{3}>$ $0 \forall k \in \mathcal{B}$ relative to (13). The third assumption $\lim _{s \rightarrow 0} s C(k ; s) P(s)=K_{v}=30\left[\mathrm{~s}^{-1}\right] \forall k \in \mathcal{B}$ is verified if for both compensators $g_{c}=300[\mathrm{~V} / \mathrm{m}]$.

The discrete minimax problem (8) has been set up with $m=5$ and $v=20$ and frequency values suitably spaced on $\Re^{+}$. In such a way the resulting problem was solved using the MATLAB optimization toolbox [17. Specifically the iterative use of the routine minimax() has permitted to determine the upper-bounds $\bar{J}_{0}, \bar{J}_{1}$, $\bar{J}_{2}$, and $M$ for each synthesized compensator. Results are the following.

1) $\mathrm{PD}$ controller

With $\bar{J}_{0}=0.016, \bar{J}_{1}=50, \bar{J}_{2}=6 \cdot 10^{5}$, and $M=1.4$ the obtained (sub-)optimal synthesis is given by $k^{*}=$ $k_{1}^{*}=0.0153$ with

$$
\begin{aligned}
& \max _{\omega \in\left[\omega^{-}, \omega^{+}\right]}\left|S\left(k^{*} ; j \omega\right)\right|=0.671, \\
& G_{m}=3.56, \quad P_{m}=40.4^{\circ},
\end{aligned}
$$$$
T_{r}=0.0231[\mathrm{~s}], \quad T_{s}=0.286[\mathrm{~s}], \quad M_{p}=2.1 \%
$$

Figures 2 and 3 2 and 3 report respectively the corresponding unit step response and magnitude plot of sensitivity.

2) third-order compensator

With $\bar{J}_{0}=0.10, \bar{J}_{1}=100, \bar{J}_{2}=7 \cdot 10^{7}$, and $M=1.4$ the (sub-)optimal synthesis is given by $k^{*}=$ $[0.001,0.001,0.0091,0.139,0.265,0.00681]^{T}$ with

$$
\begin{aligned}
& \max _{w \in\left[\omega^{-}, \omega+\right]}\left|S\left(k^{*} ; j \omega\right)\right|=0.193, \\
& G_{m}=11.6, \quad P_{m}=42.7^{\circ},
\end{aligned}
$$

$T_{r}=0.0095[\mathrm{~s}], \quad T_{s}=0.495[\mathrm{~s}], \quad M_{p}=2.6 \%(15 \mathrm{c})$

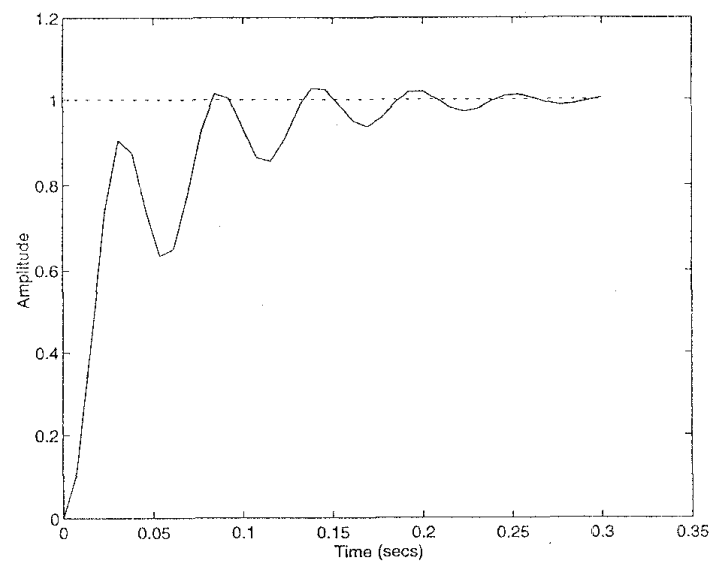

Fig. 2. Unit step response relative to $C_{1}\left(k^{*} ; s\right)$.

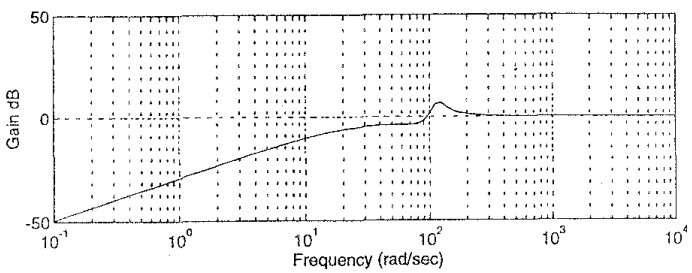

Fig. 3. Magnitude plot of $S\left(k^{*} ; j \omega\right)$ relative to $C_{1}\left(k^{*} ; s\right)$.

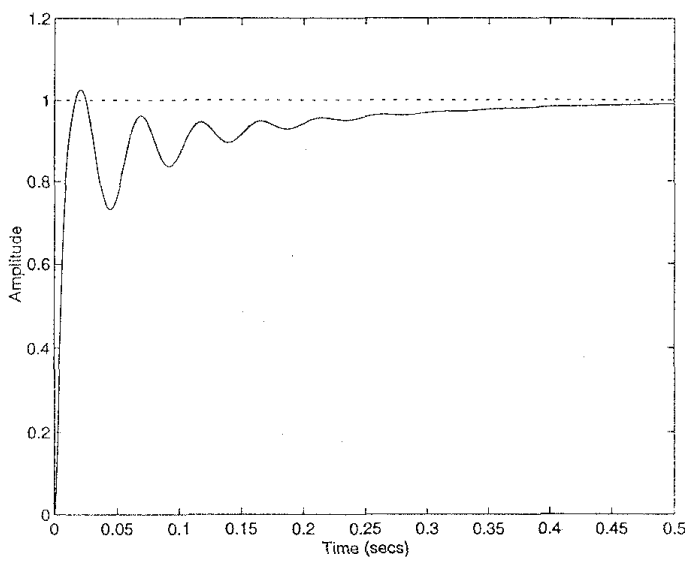

Fig. 4. Unit step response relative to $C_{2}\left(k^{*} ; s\right)$.

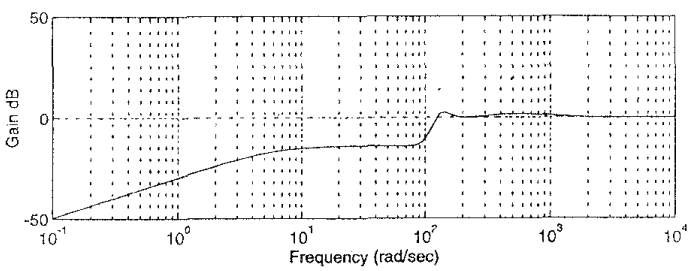

Fig. 5. Magnitude plot of $S\left(k^{*} ; j \omega\right)$ relative to $C_{2}\left(k^{*} ; s\right)$

See also Figures 4 and 5 
Remark 6: Due to the type-one transfer function of the plant the PD controller (11) operates like a PID controller.

Remark 7: In both synthesis the critical constraint is given by the specification on phase margin. Note how this harsh constraint determines in the suboptimal PD controller synthesis a poor harmonic attenuation.

Remark 8: Superiority of sub-optimal synthesis 2) over that of 1 ) is evident from results (14) and (15). Not surprisingly, as congruent with control system design experience, the sub-optimal compensator $C_{2}\left(k^{*} ; s\right)$ can almost be interpreted as that determined with an optimal zero-assignment procedure ( $\mathrm{c}$. the associated $k^{*}$ ).

\section{CONCLUSIONS}

In this paper it has been proposed an optimization method to synthesize (sub-)optimal fixed-structure compensators to attenuate harmonic disturbances over a given frequency range. The method encompasses the use of quadratic indexes of unit step tracking error to satisfy additional time-domain specifications given by upper-bounds on rise time, settling time, and overshoot. The overall procedure, which has been proved effective for small-to-medium synthesis problem, is best to be used in an interactive environment of computeraided control systems design.

From the viewpoint of optimality and robustness two are the main issues in completing this study: (i) creating ad hoc algorithms to obtain guaranteed global solutions for the synthesis problem; (ii) using an uncertain model of the plant with structured/unstructured perturbations (i.e. robustness beyond gain and phase margins). Future research will address these issues.

\section{APPENDIX}

For the reader's convenience this appendix exposes succinctly the determinantal method to compute $J_{0}=$ $\int_{0}^{\infty}[e(t)]^{2} d t$ which is due to Katz [9] and subsequently reported in [10] (see also [6, pp. 8-11]).

Let $E(s)$ denote the Laplace trasform of $e(t)$ with

$$
E(s)=\frac{B(s)}{A(s)}=\frac{b_{1} s^{n-1}+b_{2} s^{n-2}+\ldots+b_{n}}{a_{0} s^{n}+a_{1} s^{n-1}+\ldots+a_{n}} .
$$

Assume $a_{0} \neq 0$ and $A(s)$ (Hurwitz) stable for which quadratic index $J_{0}$ is well defined.

Introduce polinomial $F(s):=(-1)^{n-1}\left\{f_{1} s^{n-1}+\right.$ $\left.f_{2} s^{r-2}+\ldots+f_{n}\right\}, f_{i} \in \Re, i=1, \ldots, n$ which has to satisfy the polinomial identity:

$$
B(s) B(-s)=F(-s) A(-s)+F(s) A(s) .
$$

This relation implies that $f_{i}$ must satisfy a linear matrix equation for which, under current assumptions, an unique solution always exists; then, by virtue of Parseval's theorem, it has been proved that $J_{0}=f_{1} / a_{0}$.
Using Cramer's rule, the following closed-form expres sion of $f_{1}$ can be obtained:

$$
f_{1}=\frac{\operatorname{det}\left(\begin{array}{cccccc}
g_{1} & a_{0} & 0 & 0 & \ldots & 0 \\
g_{2} & a_{2} & a_{1} & a_{0} & \ldots & 0 \\
g_{3} & a_{4} & a_{3} & a_{2} & \ldots & . \\
. & . & . & a_{4} & \ldots & . \\
\cdot & . & . & . & \ldots & . \\
g_{n} & 0 & . & . & \ldots & a_{n}
\end{array}\right)}{\operatorname{det}\left(\begin{array}{cccccc}
a_{1} & a_{0} & 0 & 0 & \ldots & 0 \\
a_{3} & a_{2} & a_{1} & a_{0} & \ldots & 0 \\
a_{5} & a_{4} & a_{3} & a_{2} & \ldots & . \\
. & . & . & a_{4} & \ldots & . \\
. & . & . & . & \ldots & . \\
0 & 0 & . & . & \ldots & a_{n}
\end{array}\right)}
$$

where

$$
g_{i}=\frac{1}{2} \sum_{j=1}^{2 i-1}(-1)^{j-1} b_{2 i-j} b_{j} \quad\left(b_{j}=0 \text { if } j>n\right) .
$$

Since the Hurwitz determinant of order $n$-relative to $A(s)$-can be expressed as

$$
\Delta_{n}=\operatorname{det}\left(\begin{array}{cccccc}
a_{1} & a_{3} & a_{5} & . & \ldots & 0 \\
a_{0} & a_{2} & a_{4} & . & \ldots & 0 \\
0 & a_{1} & a_{3} & . & \ldots & . \\
0 & a_{0} & a_{2} & a_{4} & \ldots & . \\
. & . & . & . & \ldots & . \\
0 & 0 & 0 & . & \ldots & a_{n}
\end{array}\right)
$$

it follows that

$$
J_{0}=\frac{D_{0}}{a_{0} \Delta_{n}},
$$

with

$$
D_{0}:=\operatorname{det}\left(\begin{array}{cccccc}
g_{1} & g_{2} & g_{3} & . & \ldots & g_{n} \\
a_{0} & a_{2} & a_{4} & . & \ldots & 0 \\
0 & a_{1} & a_{3} & . & \ldots & . \\
0 & a_{0} & a_{2} & a_{4} & \ldots & . \\
. & . & . & . & \ldots & . \\
0 & 0 & 0 & . & \ldots & a_{n}
\end{array}\right)
$$

Taking into account that $\Delta_{n}=a_{n} \Delta_{n-1}$, it follows an other determinantal expression of $J_{0}$ :

$$
J_{0}=\frac{D_{0}}{a_{0} a_{n} \Delta_{n-1}}
$$

From formulae (18), (17), (19), and (16) it is apparent that $J_{0}$ is a rational expression whose arguments are the coefficients of polinomials $A(s)$ and $B(s)$.

The determinantal formula (20) can be easily generalized to compute $J_{i}=\int_{0}^{\infty}\left[D^{i} e(t)\right]^{2} d t$.

Indeed it could be proved that

$$
J_{i}=\frac{D_{i}}{a_{0} a_{n} \Delta_{n-1}}, \quad i=1,2, \ldots
$$

with $D_{i}$ being a determinant similarly defined as in (19). 


\section{ACKNOWLEDGMENTS}

The authors thank Ing. F. Tabanelli and Ing. A. Cavassi of C.N.I. Informatica S.r.I., Alfonsine (RA), Italy, for pointing out the control design problem addressed in this article.

\section{REFERENCES}

[1] P. Dufour, "Electrical drives of machine tools" (in italian), Automazione e Strumentazione, No. 10, pp. 107-119, november 1992.

[2] F. Tabanelli and A. Cavassi, "Harmonic disturbances in wood contouring machines," private communication, 1993.

[3] J.C. Doyle, B.A. Francis, and A.R. Tannenbaura, Feedback Control Theory. New York, NY: Macmillan, 1992.

[4] C.'T. Chen and C.S. Liu, "On control system design: a comparative study," IEEE Control System Magazine, Vol. 14, No. 5, pp. 47-51, October 1994.

[5] G.C. Newton Jr., L.A. Gould, and J.F. Kaiser, Analytical Design of Linear Feedback Controls. New York, NY: Wiley, 1957.

[6] J.E. Marshall, H. Górecki, K. Walton, and A. Korytowski, Time-Delay Systems: stability and performance criteria with applications. Chichester, England: Ellis Horwood, 1992.

[7] K.J. Astrom, Introduction to Stochastic Control Theory. New York, NY: Academic Press, 1970.

[8] A. Beghi, A. Lepschy, and U. Viaro, "A property of the Routh table and its use," IEEE Trans. Automat. Contr., Vol. 39, No. 12, pp. 2494-2496, 1994.
[9] A.K. Katz, "On the question of calculating the quadratic criterion for regulation," (in russian) Prikl. Mat. Mekl, Vol. 16, 362-364, 1952.

[10] E. P. Popov, The Dynamics of Automatic Control System. Reading, Mass. (U.S.A.): AddisonWesley, 1962.

[11] M. Dilda and A. Piazzi, "Using quadratic indexes in the synthesis of harmonic disturbance attenuation compensators", Dipartimento di Ingegneria dell'Informazione, Università di Parma, Report N. TSC01/95, ottobre 1995

[12] F.R. Gantmacher, The Theory of Matrices. Vol. 2. New York, U.S.A. : Chelsea, 1959

[13] M. Dilda, The Method of Quadratic Indexes in the Design of Harmonic Disturbance Attenuation Compensators (in italian), Tesi di Laurea in Ingegneria Elettronica, Università di Parma (Italy), July 1995.

[14] R. Horst and H. Tuy, Global Optimization. Berlin, Germany: Springer-Verlag, 1990.

[15] R.K. Brayton, S.W. Director, G.D. Hacrtel, and L. Vidigal. "A new algorithm for statistical circuit design based on quasi-newton methods and function splitting", IEEE Trans. Circuits and System., Vol. 26, pp. 784-794, September 1979.

[16] P.E. Gill, W. Murray, and M.H. Wright, Pratical Optimization. London, UK; Academic Press, 1981.

[17] A. Grace, Optimization Toolbox User's Guide. Natick, Mass. (U.S.A.): The MathWorks, Inc., 1994. 